\title{
Severe Edema and Venous Congestion Following Sphenoorbital Meningioma Resection in a Meningiomatosis Case: Importance of Predicting Venous Disturbances
}

\author{
Carlos Eduardo da Silva ${ }^{1}$ Alicia Del Carmen Becerra Romero ${ }^{1}$ Paulo Eduardo Peixoto de Freitas ${ }^{1}$ \\ Leonardo Desesards Olijnyk ${ }^{1}$ \\ ${ }^{1}$ Department of Neurosurgery and Skull Base Surgery, Hospital \\ Ernesto Dornelles, Porto Alegre/RS, Brazil \\ J Neurol Surg Rep 2015;76:e239-e243.

\begin{abstract}
Address for correspondence Carlos Eduardo da Silva, MD, PhD, Department of Neurosurgery and Skull Base Surgery, Hospital Ernesto Dornelles, Av. Independência 1801, Box 26, 90160-093, Porto Alegre/ RS, Brazil (e-mail: dasilvacebr@yahoo.com.br).
\end{abstract}

\begin{abstract}
Keywords

- meningioma

- cerebral veins

- sphenoparietal sinus

- dural venous sinus

- sphenoorbital

To achieve local disease control, radical removal is the best option for sphenoorbital meningiomas. Preservation of the venous system is crucial during the resection of skull base meningiomas. This vascular injury represents a major risk both for life and neurologic function. We present a case of a severe postoperative frontotemporal venous disturbance and cerebral edema following the radical removal of a giant sphenoorbital meningioma in a patient with meningiomatosis and occlusion of the superior sagittal sinus. The anatomical, radiologic, and surgical aspects are reviewed and discussed, with the aim of preventing such a serious complication.
\end{abstract}

\section{Introduction}

Large sphenoorbital meningiomas (SOMs) that compress the surrounding venous system and dural sinuses present a challenging situation in skull base surgery. Planning the surgical approach of the invasion of such vascular structures is vital to safe surgical removal. Magnetic resonance angiography (MRA) and magnetic resonance venography (MRV), including digital angiography, are used as important tools to identify the relationship between meningiomas and vessels. We present a case of SOM in a patient with meningiomatosis and occlusion of the superior sagittal sinus (SSS), with severe edema and venous congestion found during surgical removal, and discuss the aspects involved in the management of the case.

\section{Case Report}

A 51-year-old white woman presenting progressive exophthalmos, visual disturbance, and left eye pain was referred to the neurosurgical department. Magnetic resonance imaging

received

November 1, 2014 accepted after revision June 30, 2015

published online

September 14, 2015
(MRI) showed a large left SOM with surrounding edema in the temporal lobe. MRI also revealed an additional diagnosis of meningiomatosis with enhancement of the dura around the bilateral convexity and falx. A large hyperostotic left parasagittal meningioma, with total occlusion of the posterior third of the superior sagittal sinus ( - Fig. 1 ), confirmed by MRA and MRV, was also shown. The campimetry showed an abnormal visual field in the left eye. The patient had a prior history of human immunodeficiency virus and was on antiretroviral therapy. Neurofibromatosis type 2 (NF2) was excluded.

She was operated on by the first author (C.E.S.), using a left cranioorbital zygomatic approach, with aggressive removal of the SOM and extensive extradural removal of the sphenoid wing, orbital bone, and anterior temporal floor involvement (-Fig. 2A, B). Arachnoidal plane dissection of the intradural portion of the tumor was performed without any apparent arterial or venous injuries. During closure of the dura with pericranial and fascia lata grafts, a severe progressive edema developed, with considerable venous congestion and a pial
License terms

Stuttgart · New York

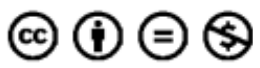

DOI http://dx.doi.org/ 10.1055/s-0035-1564058. ISSN 2193-6358. 


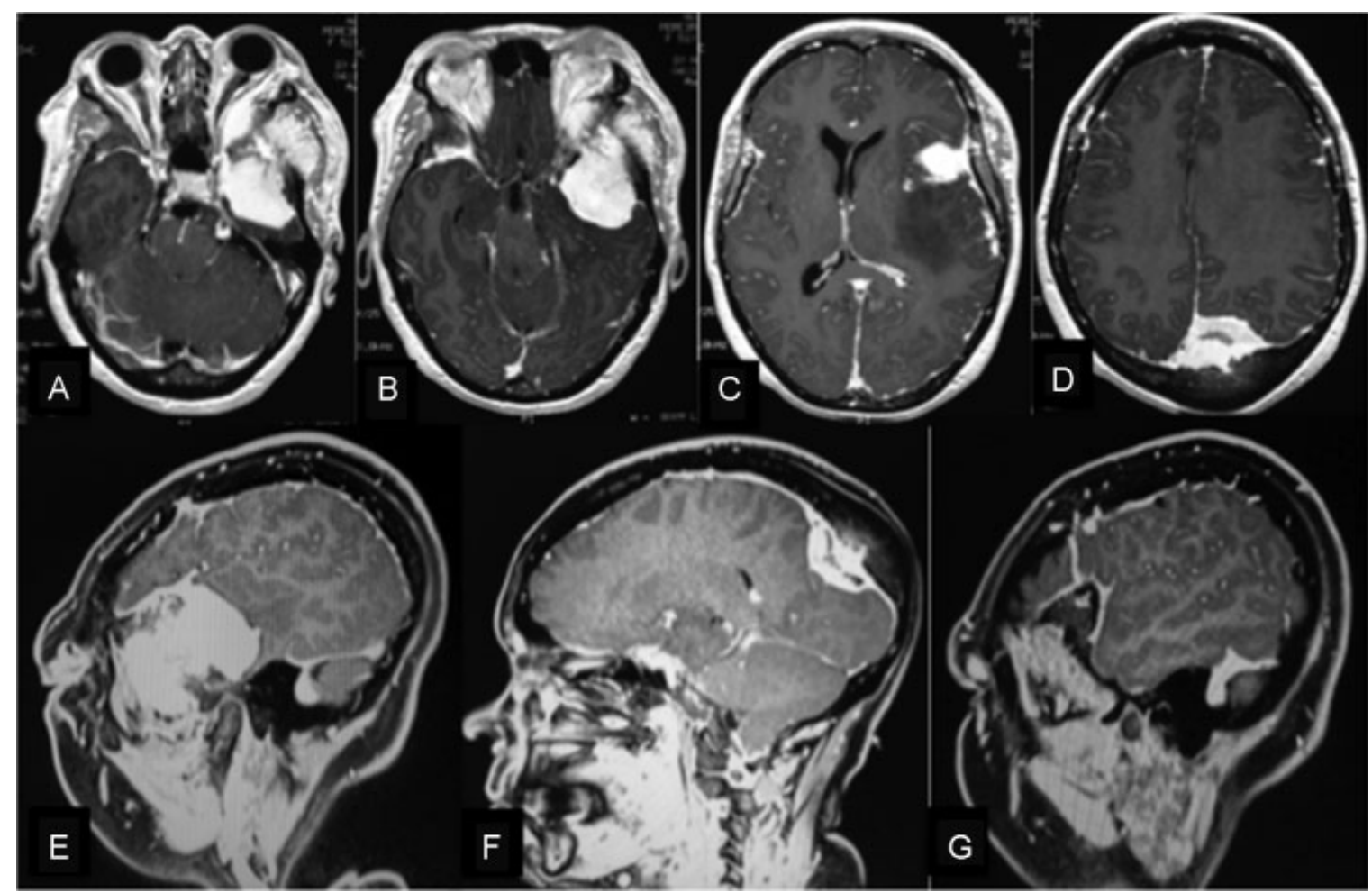

Fig. 1 Preoperative magnetic resonance imaging. (A-C) Axial images of a large left sphenoorbital meningioma and multiple meningiomas. (D) A large hyperostotic parasagittal meningioma. (E-G) Sagittal views of the sphenoorbital meningioma, parasagittal lesion, and normal sphenoorbital contralateral side.

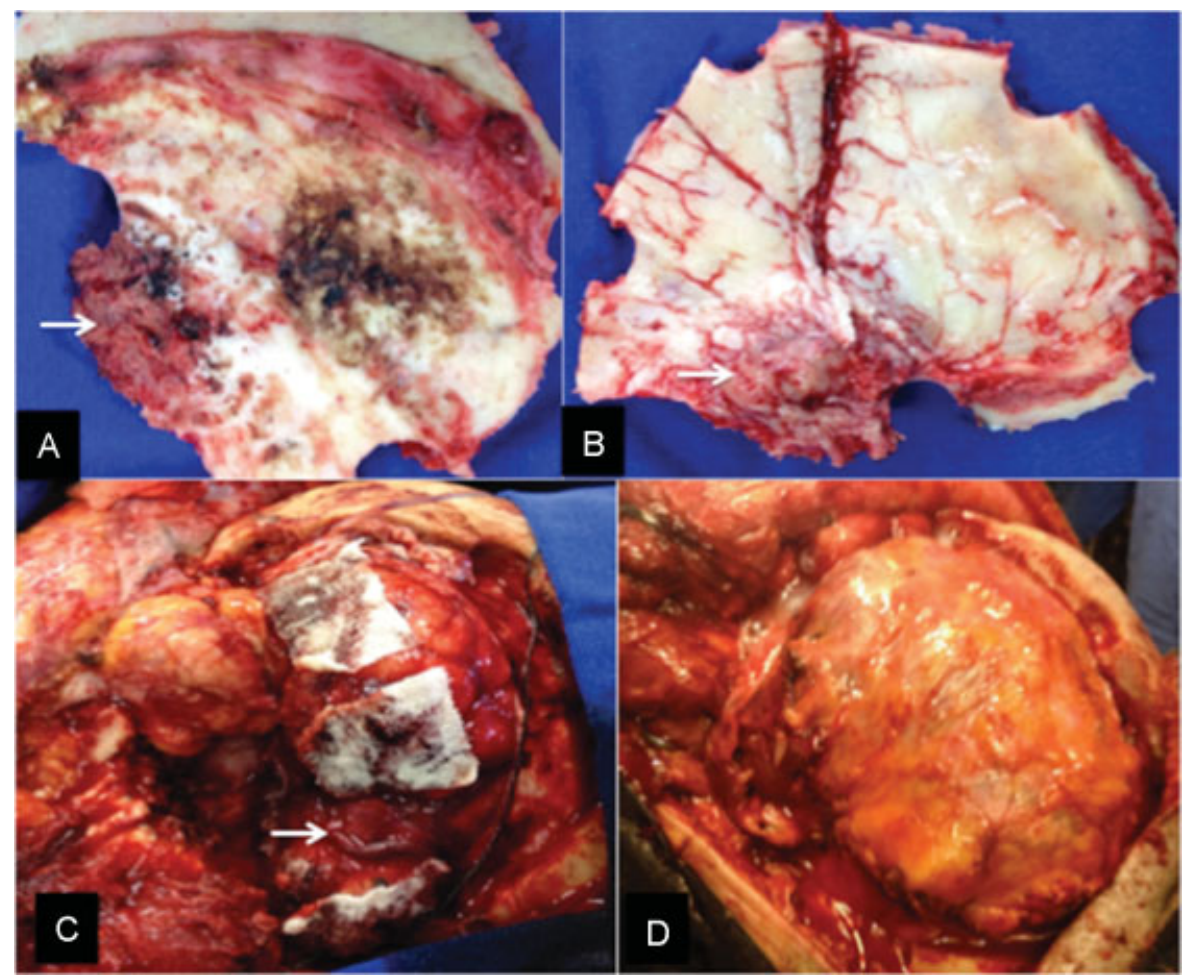

Fig. 2 Transoperative images. (A) External surface of the cranioorbital flap with hyperostotic tumoral bone (white arrow). (B) Inner surface of the bone flap and hyperostosis associated with meningioma bone invasion (white arrow). (C) Severe brain edema with pial rupture and hemostatic agents after complete removal of the left sphenoorbital meningioma and preservation of the Sylvian veins (white arrow). (D) Dural reconstruction with fascia lata and decompressive craniectomy. 
rupture (-Fig. 2C). A decompressive craniectomy was done with the insertion of intracranial pressure monitoring and intensive care unit postoperative management of intracranial hypertension. The patient developed a nominal aphasia and right-sided hemiparesis that improved during the 6 weeks that followed. Cranioplasty was performed, and the patient was discharged 8 weeks after surgery, walking with mild dysphasia. Histopathology showed a World Health Organization (WHO) grade I microcystic meningioma. Cytogenetic analysis presented a normal karyotype. A follow-up MRI, 6 months after surgery, showed satisfactory SOM removal and suggested a postoperative difference between the two frontotemporal venous drainage patterns (-Fig. 3). Meningiomatosis continues to be monitored, and the patient showed total recovery of the right-sided hemiparesis and nominal aphasia 26 months after surgery.

\section{Discussion}

Meningiomas are extra-axial lesions supplied by arterial feeders that originate from anatomical arteries responsible for the normal supply of the respective sites where tumors originate. ${ }^{1}$ Large meningiomas, WHO grade II and III meningiomas, and meningiomas with more aggressive biological behavior are also more suitable to receive pial arterial supply. Nevertheless, venous drainage is fairly variable and depends on the relationship between the tumors and the sinuses or on important drainage veins. Large meningiomas originating in the sphenoid wing, as illustrated in this case, that compress or invade important venous channels are very challenging tumors.

A giant SOM, as in the case presented here, usually compresses the superficial sylvian veins (SSVs) (-Fig. 1).

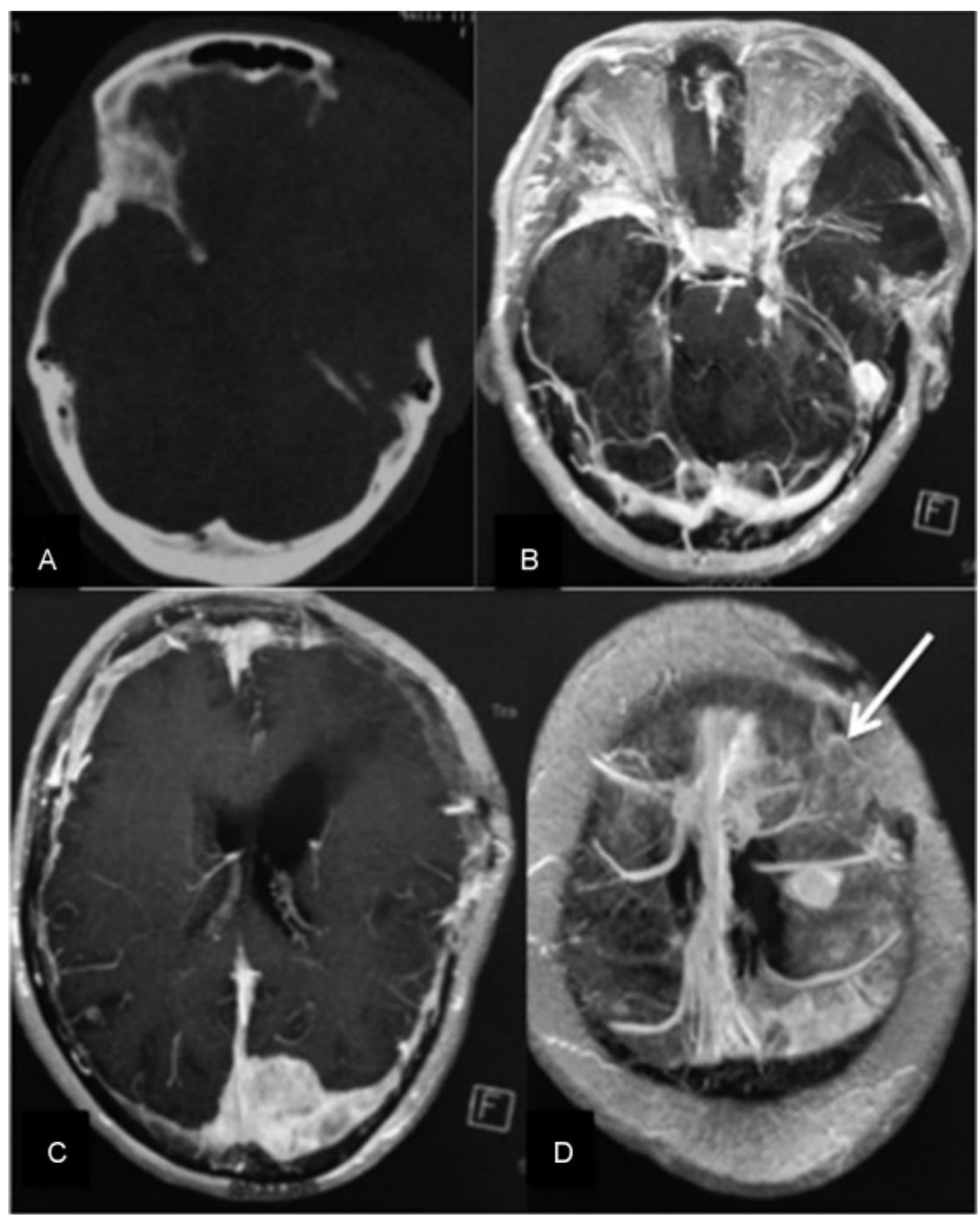

Fig. 3 Postoperative computed tomography (CT) and magnetic resonance imaging (MRI). (A) CT showing the decompressive craniectomy in the early postoperative stage. (B-D) Six-month follow-up axial MRI, after cranial reconstruction, with evidence of meningiomatosis and the aggressive removal of the left sphenoorbital meningioma. In (D) observe the difference between the venous patterns of the cerebral drainage on both sides, secondary to surgical intervention (white arrow). 
The SSV receives the frontosylvian, temporosylvian, and parietosylvian veins, coursing along the sylvian fissure and emptying into the venous channels of the sphenoid ridge or into the cavernous sinus. ${ }^{2,3}$ In addition, the veins of Trolard and Labbé are related to the SSV. The lateral frontal veins are divided in ascending and descending groups that drain to the SSS and to the SSV, respectively. ${ }^{3}$ Even so, there are innumerous configurations of such frontal veins including single or duplicated superior anastomotic veins (vein of Trolard), frontosylvian veins, and both anterior and posterior frontal veins. $^{3}$ This individual variability, associated with the obstruction in SSS secondary to the parasagittal meningioma (-Fig. 1D, F), as observed in this particular case, probably led to considerable disturbed venous drainage of the left frontal and temporal lobes.

An edema was present in the temporal lobe, posterior to the SOM, as shown on the preoperative MRI (-Fig. 1C). The edema surrounding meningiomas has provoked several hypotheses, such as a blood brain barrier disturbance, compressive effects, showing secretory aspects, pial vascularization, poor tumoral drainage veins, the size of the meningiomas, and the histology of the tumors. The preexisting edema also played an important role in the lesser complacence and in the malignant focal intracranial hypertension observed in this case. $^{4-8}$

The radical removal of the meningiomas during the first surgical approach is the most relevant aspect in the local control of the disease. Such a resection should include the tumor, dura, and hyperostotic bone. ${ }^{9,10}$ In addition, total removal of skull base meningiomas is more difficult to obtain a true Simpson grade I and grade II and achieve a lower recurrence rate. A gross total removal for skull base meningiomas, a Simpson grade III removal in most cases, means a higher recurrence rate. ${ }^{11-16}$ In a patient with non-NF2 meningiomatosis, as presented here, but who has a long life expectancy, all efforts should be made to offer radical removal and therefore better local control of the SOM.

We believe that after the resection of the meningioma that infiltrates the bone of the sphenoid ridge and during this removal of the lateral and medial portion of the dura mater underlying the sphenoid ridge, the SSV could be partially damaged. The SSV drains to the sphenoparietal sinus as it courses just below the medial portion of the sphenoid ridge and into the cavernous sinus. ${ }^{2}$ There are other possibilities of drainage of the SSV, such as the sphenobasal and sphenopetrosal sinuses, but in this particular case, the basal aspects of the temporal lobe and tentorium were not dissected. ${ }^{17}$ The cavernous sinus drainage of the SSV was completely visualized and preserved; however, the dura of the lateral and medial portion of the sphenoid wing, underlying the sphenoid ridge, was involved with the tumor and removed en bloc with the mass. The probability exists that an important venous drainage channel was interrupted at that moment, and, considering the SSS occlusion, this sudden modification was probably catastrophic for the venous net in the frontal and temporal lobes. In the minutes that followed, during closure procedures, a severe brain edema developed and a decompressive craniectomy was performed.
Some authors stress the importance of the hypoplasia of the efferent draining veins from meningiomas. They advocate that such tumor drainage plays an important role in a peritumoral brain edema. ${ }^{2}$ The patient presented considerable brain edema in the posterior temporal lobe (- Fig. 1D), and this finding could represent, as stated by these authors, poor intrinsic tumor venous drainage. To this purpose, a superselective angiography should be performed, enabling identification of the drainage pattern of the meningiomas. ${ }^{2}$ We do not consider intrinsic tumor drainage to be an explanation for the venous congestion observed in this case. Taking this hypothesis into account, tumor drainage was already poor because an edema was already present, and removal of the mass probably did not interfere with the venous balance. Moreover, such a hypothesis is interesting for large masses with occlusion of the important veins or sinuses, as in cases such as the one presented here. A superselective angiography could study the intrinsic tumor drainage and provide insight as to whether it could play a role in the venous balance. Postoperative difficulties could be anticipated and therefore better avoided.

The principal consequence of this case in our department was that the digital angiography, with venous drainage study, or dynamic MRV, is performed in all cases of meningiomas involving large veins or sinuses, despite the standard MRA or MRV. Both studies, applied to this patient, showed the relationship of the meningiomas to the arterial vessels and the occlusion of the SSS. However, they were not able to predict the dynamic of the venous drainage and the importance of the sphenoparietal sinus for venous drainage of the lateral surface of the frontal and temporal lobes. Dynamic digital angiography or MRV studies would be crucial because the anatomical information would then be associated with the hemodynamic aspects and surgery would evolve and therefore preserve these venous channels.

\section{Conclusion}

Radical removal of the sphenoorbital meningiomas is the best surgical treatment to achieve better local disease control. Digital angiography or dynamic MRV should be considered for meningiomas related to important veins and dural sinuses, despite the analysis of standard MRA and MRV, enabling safer planning of their surgical resection.

\section{References}

1 Tanaka M, Imhof HG, Schucknecht B, Kollias S, Yonekawa Y, Valavanis A. Correlation between the efferent venous drainage of the tumor and peritumoral edema in intracranial meningiomas: superselective angiographic analysis of 25 cases. J Neurosurg 2006;104(3):382-388

2 da Silva CE, Peron CS, Nesi A, Nunes CAS, Santos SC, Silveira LC. Importance of the temporal venous drainage to the petrosal approaches to the skull base. JBNC 2009;20(1):27-32. Available at: www.abnc.org.br/ed_art_down.php?id=562

3 Rhoton AL Jr. The cerebral veins. Neurosurgery 2002;51(4, Suppl): S159-S205 
4 Ide M, Jimbo M, Yamamoto M, Umebara Y, Hagiwara S, Kubo O. MIB-1 staining index and peritumoral brain edema of meningiomas. Cancer 1996;78(1):133-143

5 Lobato RD, Alday R, Gómez PA, et al. Brain oedema in patients with intracranial meningioma. Correlation between clinical, radiological, and histological factors and the presence and intensity of oedema. Acta Neurochir (Wien) 1996;138(5):485-493; discussion 493-494

6 Philippon J, Foncin JF, Grob R, Srour A, Poisson M, Pertuiset BF. Cerebral edema associated with meningiomas: possible role of a secretory-excretory phenomenon. Neurosurgery 1984;14(3): 295-301

7 Pistolesi S, Fontanini G, Camacci T, et al. Meningioma-associated brain oedema: the role of angiogenic factors and pial blood supply. J Neurooncol 2002;60(2):159-164

8 Inamura T, Nishio S, Takeshita I, Fujiwara S, Fukui M. Peritumoral brain edema in meningiomas-influence of vascular supply on its development. Neurosurgery 1992;31(2):179-185

9 Bikmaz K, Mrak R, Al-Mefty O. Management of bone-invasive, hyperostotic sphenoid wing meningiomas. J Neurosurg 2007; 107(5):905-912
10 Pieper DR, Al-Mefty O, Hanada Y, Buechner D. Hyperostosis associated with meningioma of the cranial base: secondary changes or tumor invasion. Neurosurgery 1999;44(4):742-746; discussion 746-747

11 Simpson $D$. The recurrence of intracranial meningiomas after surgical treatment. J Neurol Neurosurg Psychiatry 1957;20(1):22-39

12 Hasseleid BF, Meling TR, Rønning P, Scheie D, Helseth E. Surgery for convexity meningioma: Simpson grade I resection as the goal: clinical article. J Neurosurg 2012;117(6):999-1006

13 Obeid F, Al-Mefty O. Recurrence of olfactory groove meningiomas. Neurosurgery 2003;53(3):534-542; discussion 542-543

14 Oya S, Sade B, Lee JH. Sphenoorbital meningioma: surgical technique and outcome. J Neurosurg 2011;114(5):1241-1249

15 da Silva CE, da Silva VD, da Silva JL. Sodium fluorescein in skull base meningiomas: a technical note. Clin Neurol Neurosurg 2014; 120:32-35

16 da Silva CE, da Silva VD, da Silva JL. Convexity meningiomas enhanced by sodium fluorescein. Surg Neurol Int 2014;5:3

17 Sakata K, Al-Mefty O, Yamamoto I. Venous consideration in petrosal approach: microsurgical anatomy of the temporal bridging vein. Neurosurgery 2000;47(1):153-160; discussion 160-161 